\title{
Efectos de la valoración de los atributos del servicio de agua potable en el bienestar económico de los usuarios domésticos del área metropolitana de Huancayo
}

\author{
Effects of the attributes valuation of drinking water service on the \\ economic well-being of domestic users in the Huancayo metropolitan area
}

'Bullón García, V.

Facultad de Ciencias de la Administración, Universidad Nacional del Centro del Perú

Email: vbullon@uncp.edu.pe

\section{Resumen}

La poca valoración económica del uso del servicio de agua potable, está presente en las preferencias de las personas del área metropolitana de Huancayo (Junín, Perú). Este estudio determinó, cómo la valoración de los diversos niveles de los atributos del sistema del servicio de agua potable incide en el cambio de bienestar económico de los usuarios domésticos del área metropolitana de Huancayo.

La metodología uilizada, empleó datos de corte transversal y de diseño experimental de 400 jefes de hogar, usuarios domésticos del servicio fueron analizados, respondiendo a un cuestionario sobre elección de alternativas de mejora del servicio en relación al status quo o situación inicial en 18 sectores de distribución del servicio.

Se observó que, ante intervenciones de mejoras y disposición a pagar marginal, por cada nivel de los atributos del sistema del servicio de abastecimiento de agua potable, los usuarios domésticos aumentan su bienestar económico en S/ . 9,40 soles por mes, en constante interacción con los factores socioeconómicos como el nivel de ingresos y la edad del jefe de hogar

son necesarios estudios futuros, para seguir ganando consistencia de las relaciones causales entre los elementos del pago por servicios ecosistémicos hídricos y el diseño e implementación de políticas públicas regionales para garantizar el abastecimiento de agua potable segura.

Los diseñadores de estructuras tarifarias con pagos por servicios ecosistémicos hídricos, los evaluadores costo-beneficio de las intervenciones y los hacedores de políticas públicas regionales, apreciarán e incorporarán el valor económico de los atributos del sistema del servicio de agua potable, en el mejor estado de bienestar económico alcanzable.

Palabras clave: servicio de agua potable, servicios ecosistémicos hídricos, bienestar económico, usuarios de servicios médicos, DAP total, DAP marginal

\begin{abstract}
The low economic value of the use of drinking water service is present in the preferences of people in the metropolitan area of Huancayo (Junin, Peru). This study determined how the assessment of the different levels of the attributes of the drinking water system affects the change in the economic well-being of domestic users in the Huancayo metropolitan area.
\end{abstract}

The used methodology was cross-sectional and experimental design data for 400 household heads, domestic users of the service were analyzed and answered a questionnaire about choicing alternatives to improve the service in relation to the status quo or initial situation in 18 sectors of service distribution.

It was observed that in front of interventions of improvements and willingness to pay marginally for each level of the attributes of the drinking water service system, domestic users increase their economic well-being in S/ . 9,40 soles a month, in constant interaction with the socio-economic factors as the income level and the age of the head of household.

Future studies are necessary to keep gaining consistency in the causal relationships between the payment elements of hydric ecosystemic services and the design and implementation of regional public policies to guarantee the supply of safe drinking water.

The designers of tariff structures with payments for water ecosystemic services, the cost-benefit evaluators of interventions and the makers of regional public policies, will appreciate and incorporate the economic value of the attributes of the potable water system, in the best state of the art of economic welfare achievable.

Keywords: drinking water service, water ecosystem services, economic well-being, users of medical services, total DAP, marginal DAP. 


\section{Introducción}

Los bienes y servicios ambientales que suministran los humedales o lagunas ubicadas en las cabeceras de cuencas hidrográficas, han sido tratados como si fueran recursos gratuitos e infinitos; pero, con el crecimiento de la población y la expansión de la economía, la poca o nula planeación urbana, la falta de infraestructura, eficiencia y gestión hídrica, se han vuelto cada vez más escasos y el medio natural se ha visto cada vez más afectado. (Tudela y Soncco, 2016; BID, 2015)

Las fuentes hídricas, como manantiales, humedales y lagunas de origen pluvial o glacial, ubicadas a más de 4500 msnm en la cabecera de la sub cuenca del río Shullcas, lado este de la ciudad de Huancayo, son las principales proveedoras de agua potable a través de la empresa prestadora de servicios de saneamiento (EPS) para 379.323 habitantes del área metropolitana de Huancayo (ubicada a $3271 \mathrm{msnm}$ ), asentados en los distritos de El Tambo, $42.7 \%$; en Huancayo, $33.3 \%$ y; en Chilca, $23.0 \%$; con un crecimiento poblacional anual de 1,2\% (EPS, 2014).

La dinámica de estas ciudades, evidencian una creciente demanda de agua para consumo humano, como consecuencia de la creciente migración de la población rural y urbana de los distritos y departamentos aledaños, que según el Banco Interamericano de Desarrollo (BID, 2016) la situación se tornará insostenible para Huancayo, porque "existe el potencial de escasez del recurso en el futuro inmediato, pues quedan menos de cinco años de balance hídrico positivo".

En las últimas décadas, ante prolongadas sequías, el sistema del servicio de agua potable para los usuarios domésticos del área metropolitana de Huancayo, experimentaron niveles críticos de horas de continuidad, especialmente en zonas urbanas marginales, por debajo de siete horas por día; más aún, agravadas por la disminución de más del $50 \%$ de los volúmenes de agua almacenadas en la laguna de Huacracocha (EPS, 2014). Asimismo, se evidencia bajo conocimiento de la existencia de fuentes del agua superficial como humedales o lagunas en la cabecera de la sub cuenca del río Shullcas; demoras excesivas por más de 15 días hábiles para atención de conexiones nuevas de agua potable después de pagadas por el cliente (SUNASS, 2016; SUNASS, 2015). Por lo que, crece la necesidad de valorar desde la dimensión económica los beneficios para la sociedad a través de los diversos niveles de los atributos del sistema de servicio de agua potable, en un esfuerzo por asegurar la provisión y disponibilidad de agua potable tanto actual como en el futuro.

La valoración económica de las fuentes de agua superficiales y de la calidad del servicio de agua potable, busca capturar las preferencias sociales, preservando ciertos niveles de bienestar generados por los beneficios de su conservación y uso sostenible. Esta relación que existe entre uso y valoración, está estrechamente ligada principalmente a factores sociales y culturales (Monroy, Valdivia, Sandoval \& Rubiños, 2011).

Hjerpe \& Hussain (2016), investigaron la relación de cómo los cambios en los atributos de ecosistemas pueden conducir a cambios en la producción del servicio ecosistémico y, por qué los hogares de Alaska podrían estar dispuestos a pagar por ellos. Por lo cual, utilizaron 384 encuestas con métodos de preferencias declaradas del tipo experimentos de elección (Adamowicz, Boxall, Williams \& Louviere, 1998), concluyendo que los habitantes de Alaska tienen una fuerte preferencia con nivel de significancia del $1 \%$ por la gestión de la conservación de las cuencas hidrográficas, incluida la preservación y la restauración ecológica para las generaciones presentes y futuras. Además, Carlsson, Frykblom \& Liljenstolpe (2003), a través de experimentos de elección, identificaron dos atributos biodiversidad e instalaciones para caminar sobre el humedal Staffanstorp, al suroeste de Suecia, como los que más contribuyeron al aumento del bienestar $(\mathrm{p}<0.01)$ de los ciudadanos usuarios del humedal.

Latinopoulos (2014), realizó una investigación con pobladores griegos aplicando experimentos de elección, encontrando una buena disposición a pagar (DAP) por buena calidad de agua potable, con nivel de significancia del $1 \%$ y una menor DAP por evitar interrupciones en los servicios de agua. También, Scarpa, Thiene \& Hensher (2012), investigaron en hogares de las ciudades de Vicenza y Turín, como también en el área rural de Arzignano al norte de Italia, encontrando una importante DAP a nivel de $1 \%$ de significancia, por reducción de cuatro atributos relacionados con las características de calidad del agua potable, como: olor a cloro, sabor a cloro, turbidez del agua, manchas de carbonato de calcio en las tuberías y el costo adicional que implican las mejoras. Por otra parte, Hensher, Shore \& Train (2005), en la investigación realizada con los residentes y no residentes de la ciudad de Camberra, Australia, revelaron intentos de establecer cuánto están DAP por el atributo confiabilidad de la disponibilidad futura de agua, esto es por evitar restricciones futuras debido a sequías, de donde resulta que se promueva proyectos de inversión o incentivos a los operadores, que implica garantizar la oferta de agua en el futuro (Carbajal \& Lucich, 2016).

Con la finalidad de cubrir el vacío existente de la información en estos aspectos de valoración de la diversidad y complementariedad de los atributos naturales, económicos y operativos del sistema del servicio de agua potable en el área metropolitana de Huancayo, tal que permita incursionar en el diseño de políticas públicas y estrategias de intervención, como proyectos de infraestructura verde que aseguren principalmente la disponibilidad y continuidad de manera sostenible de dicho servicio, se planteó el objetivo de identificar y evaluar la incidencia 
de los principales atributos del sistema del servicio de agua potable, de los factores socioeconómicos relacionados y de los cambios de valoración en cada nivel de los atributos del sistema del servicio de agua potable y; en el cambio de bienestar económico de los usuarios domésticos del área metropolitana de Huancayo. A partir de ello, se puso a prueba una hipótesis que infiere de la revisión de la siguiente literatura:

H1. La valoración de los cambios en los niveles de los atributos del sistema de servicio de agua potable como: la continuidad del agua potable, la disponibilidad de la fuente del agua, la reducción del tiempo de atención de conexión de agua potable, asociados a los factores socio-económicos, inciden favorablemente en el cambio de bienestar económico de los usuarios domésticos del área metropolitana de Huancayo.

\section{Teorías de los atributos}

Lancaster (1966), argumentó que la fuente de la utilidad $U(\mathrm{Z}=B \mathrm{X})$ obtenida del consumo o uso de los bienes $\mathrm{X}$ radica en sus propiedades, características o atributos $(Z)$. A partir de los cuales, el consumo de bienes brinda utilidad indirecta V (Z, S, M-Pago), a través de las cantidades o coeficientes (B) de los diversos atributos intrínsecos que los mismos poseen, condicionadas a factores sociales $(\mathrm{S})$ y económicos como son los ingresos (M) y la Disposición Marginal a Pagar (Pago) por dichos atributos (Merino-Castelló, 2003; Holmes \& Adamowicz, 2003; Tudela \& Rodríguez, 2018; Carbajal \& Lucich, 2016; Lucich \& Gonzáles 2015).

\section{Pagos por servicios ambientales}

MINAM (2016) y MINAM (2015), precisan que, para medir el valor de los bienes y servicios ecosistémicos, como por ejemplo la regulación hídrica y la purificación del agua, se requiere relacionarlos con la variación que ellos provocan en el bienestar de los individuos o de la sociedad en el contexto donde se realiza esta interrelación; siendo el Pago o compensación por Servicios Ambientales (PSA) un mecanismo por lo que los propietarios de tierras aledañas a las fuentes de agua o lagunas recibirían a cambio de garantizar la sostenibilidad de los servicios ambientales ecosistémicos hídricos, debido a la protección contra la erosión, sedimentación y regulación de caudales (Asquith \& Wunder, 2009). Los cuales son valorados por los habitantes aguas abajo, toda vez que evidencian mejoras tanto en la continuidad y calidad del agua potable como en su utilidad o bienestar personal y colectivo (Pagiola et.A1., 2002; Landell-Mills y Porras, 2002; Pagiola y Ruthenberg, 2002), citados en Farreras (2014).

La presente investigación, pone a prueba la hipótesis 1 , bajo el supuesto de que la probabilidad de elgir una alternativa de mejoras de los atributos del sistema del servicio de abastecimiento de agua potable se ve afectada por incluir o excluir las otras alternativas de elección (Lucich \& Gonzáles, 2015; Hensher, Rose \& Greene, 2005).

\section{Materiales y métodos}

\section{Contexto}

Se consideró un marco muestral de tres zonas de distribución de agua potable: zona norte, distrito de ElTambo; zona centro, distrito de Huancayo y; zona sur, distrito de Chilca, divididos en 8 sectores, 7 sectores y 3 sectores respectivamente, estratificándose a su vez por racimos para cada uno de los 18 sectores. Las tres partes del cuestionario, fueron respondidas por cada jefe de hogar en papel, durante los meses de enero a marzo del 2018, supervisado y recogido directamente por el investigador principal responsable del proyecto. La encuesta fue anónima y hubo algunos entrevistados que manifestaban estar de prisa para con sus actividades económicas prioritarias.

\section{Participantes}

Los cuestionarios fueron respondidos por una muestra representativa de jefes de hogar seleccionados aleatoriamente de una población total 379.323 habitantes, asentados en las zonas media y baja de la subcuenca del río Shullcas, agrupados en 69.949 conexiones de usuarios domésticos del servicio de agua potable (EPS 2014), considerando un nivel de confianza del $95 \%$ para Z2 = 1,96; probabilidad de ocurrencia de las características o atributos de interés $\mathrm{p}=0,5 ; \mathrm{q}=1$-p; y error estadístico e $=4,87 \%$. La muestra incluyó a 400 jefes de hogar (Medad $=42.85, \mathrm{DS}=11,3068$ con un rango de 18 a 65 años de edad), de los cuales el $52 \%$ eran mujeres y $48 \%$ eran hombres.

\section{Mediciones}

\section{Sistema de abastecimiento.}

Se averiguó el grado de conocimiento de los problemas del sistema integral de abastecimiento, tales como fuentes de agua de la cabecera de la sub cuenca del río Shullcas, horas de servicio de agua potable, y atención de reclamos o conexiones nuevas de agua potable.

\section{Atributos y diseño experimental.}

A través de cuatro escenarios o tarjetas de elección en cada encuesta (Hensher, Rose \& Greene, 2005; Carbajal \& Lucich, 2016), se experimentaron la elección del entrevistado de las diferentes alternativas o planes de mejora en la provisión de servicios de agua potable, asociadas al pago adicional que dichas mejoras significa en el área metropolitana de Huancayo.

En términos científicos, un experimento es observar los efectos sobre una variable dependiente, dado la manipulación en los niveles de una o más variables independientes. Para dicha manipulación se recurre a formas especializadas de estadística, donde se determina qué manipulación realizar y cuándo realizar. De modo que, la manipulación ocurre por un diseño, llamado también diseño experimental. En suma, el proceso del diseño ex- 
perimental (Hensher et al., 2005; Tudela \& Leos, 2017; Tudela \& Leos, 2018; Carbajal \& Lucich, 2016); entonces, para el primer paso, se inició con el refinamiento del problema en términos de política, programas o proyectos bien definidos (mejoramiento de la continuidad del abastecimiento de agua en el hogar, recuperación de fuentes de agua para al menos 10 años y, fortalecimiento de capacidades operativas).

En el segundo paso, se identificaron cuatro atributos: continuidad de provisión del agua potable; disponibilidad de fuentes de agua; tiempo de atención de conexiones y pagos adicionales por mejoras del servicio de agua. Así mismo, se identificaron los respectivos niveles de cada atributo, sabiendo que los niveles tienen la función de describir los impactos en el atributo al cual pertenecen, esto es, aumento de continuidad de agua en el hogar a 24 horas $(\mathrm{ACO} 24 \mathrm{H})$ que equivale a un nivel Excelente o 12 horas por día $(\mathrm{ACO} 12 \mathrm{H})$ que equivale a un nivel Bueno; recuperación de la laguna de Huacracocha (RLH) Excelente o recuperación de otras lagunas (ROL) Bueno; reducción tiempo de atención conexiones nuevas a 10 días (R10D) Bueno o cinco días (R5D) Excelente; y aumento del pago mensual entre S/ . 3,00; S/ . 6,00 o; S/ . 9,00.

Así mismo, en el tercer paso, de un total de 81 combinaciones de escenarios ( $3 \times 3 \times 3 \times 3)$ o tarjetas de elección, se obtuvo nueve combinaciones a través métodos de análisis factorial fraccionado ortogonal y balanceado con el apoyo del software especializado (SPSS) con la finalidad de obtener combinaciones óptimas y viables, que minimicen la correlación entre los atributos ( Tudela \& Leos, 2017).

En el cuarto paso, se codificaron el nivel de cada variable independiente de cada atributo, con effect codes o dummy codes, con o sin interacción de las variables socioeconómicas. En efecto, cuando el entrevistado elige $\mathrm{ACO} 24 \mathrm{H}$, se asigna el valor de 1 a esta variable y 0 a la variable $\mathrm{ACO} 12 \mathrm{H}$, si por el contrario elige $\mathrm{ACO} 12 \mathrm{H}$, entonces se asigna a esta variable el valor de 1 y 0 a la variable $\mathrm{ACO} 24 \mathrm{H}$; la última opción es que el entrevistado prefiera "Deficiente", en este caso se codifica con -1 a la variable $\mathrm{ACO} 24 \mathrm{H}$ y también con -1 a la variable ACO12H (siendo 0 en ambas variables si se usa dummy codes). Los coeficientes de ACO24H y ACO12H proveen la "utilidad marginal" de esos niveles del atributo continuidad de agua, multiplicando por -1 la suma de esos coeficientes se puede obtener la "utilidad marginal" del nivel "Deficiente" de continuidad de agua. Los códigos para los otros dos atributos: Disponibilidad de fuentes de agua y; tiempo de atención de conexiones, se codificaron similarmente.

El quinto paso, se refiere a la asignación de columnas dentro del diseño para la generación del conjunto de elección, se recomienda no manipular el diseño y no ge- nerar una respuesta estimulada. El sexto paso, implica la generación de conjuntos de elección que son utilizados en la encuesta. En el séptimo paso, las ocho combinaciones óptimas que se generaron en el diseño ortogonal se combinaron aleatoriamente en grupos de dos, añadiendo una tercera alternativa en cada conjunto de elección que representa la situación actual (statu quo) o nivel Deficiente de pago adicional nulo y no genera ningún cambio respecto a la situación actual.

El último paso, del proceso de diseño experimental, constituye la construcción de las tarjetas de elección, el mismo que se incluye en el formato de encuesta. En particular, como se generaron cuatro tarjetas de elección (Tarjeta 1 , tarjeta 2 , tarjeta 3 y tarjeta 4 ) con tres atributos del servicio de agua y tres niveles (excelente, bueno, deficiente), se evidenció un experimento con cuatro repeticiones, de ese modo se obtuvo una base de datos tipo datos de panel, con 4 x $3=12$ observaciones efectivas por cada entrevistado, distribuidas en 400 encuestas, 1.600 elecciones efectivas ( $400 \times 4)$, totalizando 4.800 observaciones ( 4 x 3 x 400). Es necesario recalcar que, el proceso del diseño experimental puede impactar significativamente la eficiencia de las estimaciones de las DAP, que juegan un papel central en la valoración de servicios de no de mercado (Scarpa, Thiene \& Hensher, 2012).

\section{Factores socioeconómicos.}

En la parte tres de la encuesta, a través de ocho preguntas, se procuró captar información socioeconómica relevante con datos cuantitativos o cualitativos del entrevistado.

\section{Modelos multinomiales.}

Los modelos logit multinomial y modelo logit mixto (que contiene parámetros fijos y aleatorios), precisan que la variable dependiente bienestar es la elección que realiza el entrevistado sobre la base de alternativas o planes de mejora de los atributos del servicio de abastecimiento de agua potable, de ahí que se trabajó con dos variables de cada atributo en las que el entrevistado puede elegir cualquiera de dos niveles de mejora (Excelente o Bueno) o del statu quo (Deficiente). Así mismo, las variables explicativas que corresponden a los diferentes niveles de mejora de los atributos del servicio de agua potable, asociados con los factores socioeconómicos en su condición de variables extrañas o intervinientes de los entrevistados, son codificadas con effect codes o dummy codes y se especifican de manera aditiva o con interacciones.

Por lo anterior, se especificó la forma funcional lineal del modelo de utilidad aleatoria (McFadden, 1974; Carbajal \& Lucich, 2016):

Sin interacciones: 
$U_{i j}=\beta 1 \mathrm{ACO} 24 \mathrm{H} i j+\beta 2 \mathrm{ACO} 12 \mathrm{Hij}+\beta 3 \mathrm{RLH} i j+$ $\beta 4 \mathrm{ROL} i j+\beta 5 \mathrm{R} 5 \mathrm{D} i j+\beta 6 \mathrm{R} 10 \mathrm{D} i j-\beta 7 \mathrm{PAGO} i j+\varepsilon i j$

(1)

Con interacciones:

$U_{i j}=\beta 1 \mathrm{ACO} 24 \mathrm{H} i j+\beta 2 \mathrm{ACO} 12 \mathrm{H} i j+\beta 3 \mathrm{RLH} i j+$ $\beta 4 \mathrm{ROL} i j+\beta 5 \mathrm{R} 5 \mathrm{D} i j+\beta 6 \mathrm{R} 10 \mathrm{D} i j-\beta$ PPAGO $i j+$ $\beta$ 8GENEROX $i j+\beta$ 9NINTEGX $i j+\beta 10$ EDADX $i j+$ $\beta 11 \mathrm{EDUCX} i j+\beta 12 \mathrm{ACTIV} X i j+\beta 13 \mathrm{INGR} X i j+\varepsilon i j$

Donde:

$U i j$ : utilidad directa del individuo i al elegir la alternativa $j$; $\beta$ s: son valores marginales o coeficientes de cada nivel de atributo; $\varepsilon_{-} \mathrm{ij}$ : error aleatorio; $\mathrm{ACO} 24 \mathrm{H}$ : continuidad del agua potable durante 24 horas por día; ACO12H: continuidad del agua potable durante 12 horas por día; RLH: recuperación y conservación de la laguna de Huacracocha; ROL: recuperación y conservación de otras lagunas; R5D: reducción en el tiempo de atención a 5 días; R10D: reducción en el tiempo de atención a 10 días; PAGO: importe adicional por alternativas de mejoras en cada nivel de atributo; GÉNEROX: Masculino o femenino (1 o 2); NINTEGX: Número de integrantes por hogar; EDADX: Edad en años; EDUCX: Educación $(1,2, \ldots, 10)$; ACTIVX: Actividad económica $(1,2, \ldots, 6)$; INGRX: Ingreso familiar promedio mensual en soles; una variable dummy $(\mathrm{X})$, que distingue entre las alternativas de mejora (1), sobre la alternativa específica del status quo (0); y $E i j$ : término estocástico o error aleatorio (idéntica e independientemente distribuido) (iid).

De la familia de modelos logísticos de probabilidad, como: multinomial o condicional (McFadden, 1974; Louviere, Hensher \& Swait, 2000), y el mixto o de parámetros aleatorios, este último es un modelo más avanzado debido a su capacidad para modelar la heterogeneidad en las preferencias de los individuos mediante el empleo de parámetros aleatorios, permitiendo variación aleatoria de preferencias, patrones de sustitución no restringidos y correlación entre factores no observados a lo largo del tiempo; por lo que, se supera el supuesto de "independencia de las alternativas irrelevantes" (IIA) (Hausman \& MaFadden, 1984; Hensher et al., 2005; Tudela \& Leos, 2017). Es decir, los coeficientes de cada nivel de los atributos varían aleatoriamente entre los usuarios, generando estimaciones más robustas y confiables.

Las estimaciones de los modelos logit multinomial y mixto, con o sin interacción, con codificación effect codes y dummy codes de los niveles de cada atributo, se basan en los métodos de máxima verosimilitud sin o con simulación respectivamente, a efectos de estimar asintóticamente el máximo valor de los parámetros de cada variable explicativa y de las variables intervinientes.
A su vez, para cada modelo se evalúa el signo esperado positivo o negativo de los coeficientes de cada nivel de los cuatro atributos, el valor de cada coeficiente, su nivel de significancia estadística " $t$ ", su bondad de ajuste global a través del estadístico Razón de Verosimilitud-log likelihood, y su poder explicativo del R2 de McFadden, para saber qué modelo se ajusta mejor a los datos obtenidos. Así mismo, para la significancia conjunta del modelo, se utilizó la Chi cuadrada con 18 grados de libertad y un valor "p" igual a cero, esto es, se testea la hipótesis nula que todos los betas sean iguales a cero, rechazándose dicha hipótesis (Carbajal \& Lucich, 2016).

\section{Bienestar económico.}

La estimación de las medidas monetarias del bienestar y el cálculo del efecto en el bienestar, exige en primer lugar, estimar la tasa marginal de sustitución entre dos atributos como el cociente de sus coeficientes o parámetros, generando la estimación de la disponibilidad a pagar marginal (DAPMg) por un cambio en el atributo $Z_{k}$, dado por (Alpízar, Carlsson, \& Martinsson, 2001; Tudela \& Leos, 2017) por la ecuación (3):

$$
D A P M g_{\alpha}=\frac{\partial v_{i j} / \partial Z_{\alpha}}{\partial v_{i j} / \partial P A G O}=-\frac{\beta_{\alpha}}{\gamma}
$$

Donde: $\beta \alpha$ es el coeficiente del atributo del cual se desea medir la disposición marginal a pagar, y $\mathrm{y}$ es el coeficiente relativo a los ingresos de la persona o al precio adicional del atributo alternativo. En segundo lugar, se estima el cambio en el bienestar económico a través de la variación compensatoria (VC), teniendo en cuenta escenarios de mejora, que en versión simplificada (Tudela \& Leos, 2017) queda expresada como sigue:

$$
V C=\left[\frac{1}{-\gamma}\right]\left(v_{i 1}-v_{i 0}\right)=\frac{\beta_{k}}{-\gamma}\left(Z^{1}-Z^{0}\right)
$$

Dónde: donde v_i1 representa la utilidad del nivel de mejora, $v_{i 0}$ la utilidad del statu quo y $\gamma$ la utilidad marginal del ingreso (PAGO).

\section{Estrategia analítica.}

Después de confirmar que los cuestionarios son fiables y adecuados para ser utilizados en la muestra estimada, la base de datos se sistematizó con el Excel.Y los estadísticos descriptivos, la matriz de correlación, los coeficientes o parámetros de los modelos econométricos a través del método de máxima verosimilitud simulada, la distribución de Kernel, y los intervalos de confianza de la DAP con método Delta y procedimiento de Krinsky y Robb, se calcularon con el software NLOGIT 4.0 (Tudela \& Leos 2017). 


\section{Resultados}

\section{Análisis preliminares}

Problemas del abastecimiento de agua potable.

Se infiere que, sólo el $14 \%$ de los usuarios manifestaron conocer la existencia de la laguna de Huacracocha, $5 \%$ de la laguna de Lazo Huntay y $1 \%$ de la laguna de Chuspicocha; no obstante, que existen siete lagunas más administradas por la EPS. Así mismo, 7 \% conocen el punto de captación $\mathrm{N}^{\mathrm{o}} 24$ del agua natural superficial de la sub cuenca del río Shullcas ubicado a dos $\mathrm{km}$ al noreste de la Planta de Tratamiento de Agua Potable de Vilcacoto, sector de "Chamisería”. En contraste con el 73 \% que no conoce algunas fuentes de agua superficial.

Además, el $98 \%$ de los usuarios manifestaron haber experimentado, al menos una vez, interrupciones durante los últimos tres meses en el servicio de agua potable por motivos de rupturas o atoros en horarios de continuidad del servicio de agua. Por el contrario, solo $2 \%$ no precisa haber tenido ninguna interrupción.

Por otro lado, el $58 \%$ de los usuarios manifestaron problemas de calidad del agua potable durante los tres últimos meses, sea por turbiedad, olor o algas; consecuencia de la erosión del suelo por actividades agrícolas, ganaderas, de construcción y piscícolas, de comunidades asentadas en la zona media y zona alta de la sub cuenca del río Shullcas. También, el $53 \%$ de los usuarios percibieron que la mala calidad del agua afecta de manera importante la salud de la familia, manifestada por males estomacales o diarreas agudas, sobre todo en los niños y ancianos.

Más aún, el $50 \%$ de los usuarios, declararon que el atributo más importante del servicio de agua potable es la disponibilidad del agua potable durante las 24 horas del día en sus viviendas $(r=0,18)$, seguido del $46 \%$, que la EPS debe asegurar las fuentes del agua ubicadas en la cabecera de la subcuenca del río Shullcas, específicamente la laguna de Huacracocha $(r=0,21)$. En tanto que el tiempo de atención y reclamos en conexiones nuevas de agua potable a sus viviendas representaron el $27 \%$ de las respuestas de los entrevistados $(r=0,18)$. En cambio, el $16 \%$ de los usuarios manifestaron que el servicio de agua potable es aceptable; en tanto, que para el $84 \%$, el servicio es regular o deficiente.

\section{Información socioeconómica.}

El $52 \%$ de los usuarios fueron del sexo femenino, relacionándose en forma negativa con su nivel educativo $(r=-0,19)$ y con el ingreso familiar $(r=-0,22)$. Así mismo, para el $68 \%$ de los usuarios, el número de integrantes por hogar fue entre 3 a 5 integrantes (hijos, padres, hermanos) (Media $=4,74, \mathrm{DS}=1,53$, Mínimo $=1$, Máximo $=9$ ), y se relaciona débil y negativamente con la educación $(r=-0,04)$. Se debe agregar que, el 30\% de los usuarios manifestaron tener entre
36 a 45 años de edad (Media $=42,85$, DS $=11,31$, Mínimo $=18$, Máximo $=65)$, relacionándose negativamente $(r=-0,11)$ con el ingreso familiar. También, el $27 \%$ de los usuarios evidenciaron estudios universitarios, en tanto que el $16 \%$ nivel superior técnica, relacionándose positivamente con diversas actividades económicas $(r=0,31)$ como: comercio, servicios, transporte, entre otros.

Por otra parte, la variable ingreso familiar se ubicó mayormente $(26,3 \%)$ entre S/ 1.500 a S/. 2.500 mensuales $($ Media $=\mathrm{S} /$. 2.102, $\mathrm{DS}=\mathrm{S} /$. 1.163, Mínimo $=\mathrm{S} /$. 400,00 Máximo $=\mathrm{S} / .7 .500)$, mostrando una correlación positiva con el nivel educativo $(r=0,42) \mathrm{y}$ las actividades económicas $(r=0,23)$. Además, la actividad comercial resulto como la más importante para el $38 \%$ de los usuarios, seguida por la actividad en el sector público con el $20 \%$, y por la actividad de servicios con el $19 \%$.

\section{Experimento de elección.}

En la encuesta, al presentarles un conjunto de alternativas para la elección de los usuarios, 746 (47\%) de ellos; eligieron la alternativa A (Tabla 1); 544 (34 \%) usuarios, eligieron la alternativa B y; 310 (19\%) usuarios, eligieron la alternativa $\mathrm{C}$ que es el statu quo; esto es, un $81 \%$ de los usuarios, prefirieron mejoras en los atributos del servicio de agua potable.

Tabla 1

Preferencias de alternativas de elección por tipo de tarjetas

\begin{tabular}{lcccc}
\hline \multirow{2}{*}{ Tipo * } & \multicolumn{3}{c}{ Alternativas } & Total \\
\cline { 2 - 4 } & $\mathbf{A}$ & $\mathbf{B}$ & $\mathbf{C}$ & \\
\hline Tarjeta 1 & 257 & 116 & 27 & 400 \\
Tarjeta 2 & 173 & 136 & 91 & 400 \\
Tarjeta 3 & 242 & 128 & 30 & 400 \\
Tarjeta 4 & 74 & 164 & 162 & 400 \\
Total & 746 & 544 & 310 & 1600 \\
\hline
\end{tabular}

* Conjunto de tarjetas de elección, donde cada tarjeta contiene cuatro atributos, dos variables y tres niveles: continuidad de agua potable (aumento a 24 horas, aumento a 12 horas); disponibilidad de fuentes de agua (recuperación de laguna de Huacracocha, recuperación de otras lagunas); tiempo de atención de conexiones (reducción a 5 días, reducción a 10 días) y; pagos adicionales de S/.3.0; S/ . 6.0; S/.9.0 mensuales. Así mismo los niveles: Excelente, Bueno y Deficiente. Distribuidas aleatoriamente en cada alternativa A, B, C; debido al uso del diseño factorial fraccionado ortogonal y balanceado, y los effects codes.

Es necesario recalcar que, después de varias especificaciones y estimaciones, se eligió el modelo logit mixto (Tabla 2), con interacción de dos variables socioeconómicas y con effect codes, resultando el siguiente modelo (5): 
$\mathrm{V}_{\mathrm{ij}}=0.762 \mathrm{ACO} 24 \mathrm{H}+0.202 \mathrm{ACO} 12 \mathrm{H}+$ $0.168 \mathrm{RLH}-0.293 \mathrm{ROL}+0.797 \mathrm{R} 5 \mathrm{D}+0.319 \mathrm{R} 10 \mathrm{D}$ - 0.204PAGO-0.28 (1 $\left.1_{\text {EDAD }}\right)+0.0008(1$ INGR) $0.031\left(2 \_E D A D\right)+0.0008\left(2 \_\right.$INGR $)$

En efecto, los signos de los parámetros aleatorios asociados al atributo continuidad del agua potable $(\mathrm{ACO} 24 \mathrm{H}$, ACO12H), fuente de agua (RLH), tiempo de atención de conexión (R5D, R10D), costo adicional (PAGO) y del parámetro no aleatorio del ingreso familiar mensual (INGR), son los esperados según la teoría; sin embargo, los parámetros de la fuente de agua (ROL) y edad (EDAD), presentan signos contrarios a lo que podría esperarse, indicando que los usuarios no desean que la fuente de agua recuperación de otras lagunas sea una opción, más aún, si ostentan una mayor edad.

Tabla 2

Modelos logit multinomial y logit mixto

\begin{tabular}{|c|c|c|c|c|}
\hline \multirow[b]{2}{*}{ Variables } & \multicolumn{2}{|c|}{ Logit multinomial } & \multicolumn{2}{|c|}{ Logit mixto } \\
\hline & $\begin{array}{l}\text { Effect } \\
\text { codes }\end{array}$ & $\begin{array}{c}\text { Dummy } \\
\text { codes }\end{array}$ & $\begin{array}{l}\text { Effect } \\
\text { codes }\end{array}$ & $\begin{array}{c}\text { Dummy } \\
\text { codes }\end{array}$ \\
\hline \multirow{2}{*}{$\mathrm{ACO} 24 \mathrm{H}$} & 0.658 & 1.432 & 0.76226543 & 1.776 \\
\hline & $(8.276)^{* * *}$ & $(6.859)^{* * *}$ & $(5.527)^{* * *}$ & $(4.15)^{* * *}$ \\
\hline \multirow{2}{*}{$\mathrm{ACO} 12 \mathrm{H}$} & 0.115 & 0.889 & 0.20223415 & -0.671 \\
\hline & (1.407) & $(4.201)^{* * *}$ & $(1.405)$ & $(-0.439)$ \\
\hline \multirow{2}{*}{ RLH } & 0.213 & 0.113 & 0.16839288 & 1.419 \\
\hline & $(2.277)^{* *}$ & $(0.565)$ & (1.154) & $(1.151)$ \\
\hline \multirow{2}{*}{ ROL } & -0.314 & -0.414 & -0.29276855 & -0.937 \\
\hline & $(-3.283)^{* * *}$ & $(-2.039)^{* *}$ & $=(-2.589) * *$ & $(-1.485)$ \\
\hline \multirow{2}{*}{ R5D } & 0.703 & 1.72 & 0.79722914 & 2.901 \\
\hline & $(7.747)^{* * *}$ & $(9.973)^{* * *}$ & $(5.356)^{* * *}$ & $(2.914)^{* * *}$ \\
\hline \multirow{2}{*}{ R10D } & 0.313 & 1.33 & 0.31895842 & 3.408 \\
\hline & $(2.923)^{* *}$ & $(6.692)^{* * *}$ & $2.705)^{* * *}$ & $(1.75)^{*}$ \\
\hline \multirow{2}{*}{ PAGO } & -0.173 & -0.174 & -0.20364114 & -0.461 \\
\hline & $(-6.699)^{* * *}$ & $(-6.699)^{* * *}$ & $*(-4.465)^{* * *}$ & $(-1.84)^{*}$ \\
\hline \multirow{2}{*}{ 1_EDAD } & -0.026 & -0.026 & -0.02824504 & -0.034 \\
\hline & $(-3.871)^{* * *}$ & $(-3.871)^{* * *}$ & $*(-3.845) * * *$ & $(-3.55)^{* * *}$ \\
\hline \multirow{2}{*}{ 1_INGR } & 0.0007 & 0.0007 & 0.00077878 & 0.0009 \\
\hline & $(8.542)^{* * *}$ & $(8.542)^{* * *}$ & $(8.118)^{* * *}$ & $(7.542)^{* * *}$ \\
\hline \multirow{2}{*}{ 2_EDAD } & -0.029 & -0.029 & -0.03051559 & -0.027 \\
\hline & $(-4.829)^{* * *}$ & $(-4.829) * * *$ & $*(-4.607) * * *$ & $(-3.619)^{* * *}$ \\
\hline \multirow{2}{*}{ 2_INGR } & 0.0007 & 0.0008 & 0.00081663 & 0.0008 \\
\hline & $(9.119)^{* * *}$ & $(9.119)^{* * *}$ & $(8.03)^{* * *}$ & $(7.901)^{* * *}$ \\
\hline Log-likelihood & -1451.978 & -1451.978 & -1449.741 & -1447.478 \\
\hline Chi-squared & & & 616.077 & 620.6032 \\
\hline Pseudo R-squared & 0.12678 & 0.12678 & 0.17524 & 0.1765304 \\
\hline Pseudo R-squared Adj. & 0.12376 & 0.12376 & 0.17058 & 0.17187 \\
\hline Nro. de observaciones & 4800 & 4800 & 4800 & 4800 \\
\hline
\end{tabular}

Nota. Entre paréntesis “ $\mathrm{t}$ ”-estadísticos: $* * *$ indica significancia a un nivel de $1 \%$; * al $5 \%$; y al $10 \%$

\section{Prueba de la hipótesis del estudio}

El modelo logit mixto con interacciones effect codes (Tabla 2), evidencia alta significancia conjunta del modelo (Chicuadrada $=616,077$ con 18 grados de libertad y un valor $p=0,00)$, una buena bondad de ajuste global (Razón de verosimilitud $=-1449,741$ ) y aceptable poder explicativo (R2 de McFadden = 0,17058), permitiendo rechazar la hipótesis nula de que todos los coeficientes $(\beta s)$ sean iguales a cero o que no hay relación entre los cambios en los niveles de cada atributo y los factores socioeconómicos relacionados, y el cambio de bienestar de las personas del área metropolitana de Huancayo.

Es decir, a mayor nivel de los parámetros aleatorios de las variables ACO24H, R5D, R10D, y del parámetro no aleatorio del INGR, el bienestar de los usuarios $\left(V_{i j}\right)$ aumenta de manera significativa $(\mathrm{p}<0,01)$ y a mayor nivel de los parámetros alaeatorios y no aleatorios asociados a las variables ROL, PAGO, y EDAD respectivamente, este bienestar disminuye significativamente $(\mathrm{p}<0,01)$.

Por otra parte, no se encontró una relación significativa entre los atributos $\mathrm{ACO} 12 \mathrm{H}$, fuentes de agua RLH y el bienestar $(p=0,16)$, aunque en el modelo logit multinomial sin interacción, esta relación es positiva y significativa $(\mathrm{p}<0,05)$.

En particular, las diferencias de elección entre los usuarios se explicaron por las diferencias de edad, por el ingreso y por el pago mensual del servicio. En cambio, el género, el número de integrantes y la actividad económica del jefe de hogar no explican estas diferencias. En los modelos logit multinomial con interacción de todas las variables socioeconómicas, se evidenciaron indicios de significancia del nivel educativo de los usuarios.

\section{Discusión}

El exiguo conocimiento de los atributos (Lancaster, 1966) del servicio de abastecimiento de agua potable determinan un bajo valor de mercado del agua (Iglesias, 2017), repercutiendo en la cantidad, calidad y oportunidad de dicho servicio, con los efectos de insatisfacción, malestar y reclamos, afectando la salud de las personas (Justes, Barberán \& Farizo, 2014) y, por consiguiente, el bienestar individual y colectivo.

Desde el lado económico, la medición y valoración de los diferentes atributos del servicio de agua potable (Scarpa, et al., 2012; Ojeda De La Cruz, et al., 2017; Carbajal \& Lucich, 2016), que incluyeron la recuperación y conservación de fuentes de agua (Carlsson, et al., 2003; Hjerpe \& Hussain, 2016; Tudela \& Soncco, 2016; Cerda, 2011; Monroy, et al., 2011), se vienen desarrollando e implementando en diversas partes del mundo sobre todo en zonas vulnerables al cambio climático, a través de experimentos de elección, con la finalidad de explicar los cambios en el bienestar de las personas. Sin embargo, 
en países como el Perú, existen muchas lagunas en el conocimiento de los diversos atributos como fuentes de agua, factores operativos, y de los factores socioeconómicos y culturales (Koehler, Rayner, Katuva, Thomson \& Hope, 2018), cuya comprensión integral y sistémica promete seguridad hídrica (Sadoff \& Muller, 2010) para el consumo humano, fundamental para la salud humana, con la consiguiente mejora del bienestar de las personas.

Por lo que, para revertir el deterioro del sistema de abastecimiento del agua potable en el área metropolitana de Huancayo, es importante considerar no solo la tecnología costo-efectiva, sino las preferencias declaradas de los usuarios domésticos con respecto a los atributos de dicho servicio.

De los 1.600 experimentos aplicados, el 81\% de los usuarios eligieron algunas alternativas o planes de mejora del conjunto de atributos y sus respectivos niveles, aceptando pagar un monto adicional mensual en su recibo de agua; mientras que el 19\% prefirió mantenerse en su situación actual (status quo) y no pagar ningún monto adicional. Estos resultados se encuentran en línea con los encontrados por Tudela \& Leos (2017); Lucich \& Gonzáles (2015).

La disponibilidad a pagar marginal (DAPMg), por cambios en cada nivel de los atributos del servicio de agua potable (Tabla 3), se estimaron en: S/ . 3,74 por 24 horas día de continuidad del agua potable, sobre todo por quienes habitan en sectores del área metropolitana de Huancayo, donde la continuidad está por debajo de siete horas por día (de un total de 24 horas día) agravada en épocas de sequías prolongadas, con una tendencia decreciente por la que SUNASS (2016) calificó a la EPS como de desempeño "Malo". Así mismo, se evidencian pagos adicionales de S/ . 0,99 por 12 horas de servicio día; $\mathrm{S}$ /. 3,91 por reducción a 5 días del tiempo de atención por conexiones nuevas; $\mathrm{S} /$. 1,57 por reducción a 10 días en tiempo de atención conexión nueva; indicios de S/ . 0,83 por recuperar y conservar la laguna de Huacracocha.

Tabla 3

Disponibilidad a pagar marginal por un cambio en nivel por cada atributo

(Soles/mes/vivienda)

\begin{tabular}{lcccc} 
& \multicolumn{2}{c}{ (Soles/mes/vivienda) } & \\
\cline { 2 - 3 } $\begin{array}{c}\text { Servicios de agua } \\
\text { potable (atributos) }\end{array}$ & $\begin{array}{c}\text { DAPMg por niveles } \\
\text { de mejora } *\end{array}$ & $\begin{array}{c}\text { DAP } \\
\text { Total }\end{array}$ & \\
\cline { 2 - 3 } & Bueno & Excelente & \\
\hline $\begin{array}{l}\text { Continuidad del } \\
\text { agua potable }\end{array}$ & 0.99 & 3.74 & 4.74 & $49 \%$ \\
$\begin{array}{l}\text { Disponibilidad } \\
\text { fuente del agua }\end{array}$ & -1.44 & 0.83 & -0.61 & $-6 \%$ \\
$\begin{array}{l}\text { Tiempo de atención } \\
\text { conexiones nuevas }\end{array}$ & 1.57 & 3.91 & 5.48 & $57 \%$ \\
\hline \begin{tabular}{l} 
Total \\
\hline
\end{tabular} & 1.12 & 8.48 & 9.61 & $100 \%$ \\
\hline
\end{tabular}

(*) Se acotó en intervalos de confianza al $95 \%$ mediante los métodos los métodos Delta y K\&R.
Por consiguiente, al agregar todos los valores marginales se estimó un valor total de $\mathrm{S} /$. 9,61 por usuario mes/vivienda, como pago adicional al consignado en su recibo, debido a la mejora de los atributos del servicio de abastecimiento de agua potable; distribuyendo un $49 \%$ del incremento en la tarifa, para asegurar la prestación del servicio de agua potable durante las 24 horas día; un 57 \%, para mejorar la gestión operativa de la EPS, en cuanto al tiempo de atención de reclamos o conexiones nuevas y; un $6 \%$, para compensar la pérdida de bienestar que significaría traer agua de otras lagunas distintas a la laguna de Huacracocha.

Por otra parte, los planes de mejora también incorporan el atributo fuentes de agua, por lo que, los usuarios revelaron que recuperar y conservar la laguna de Huacracocha significa un aporte adicional de $\mathrm{S} / .0,83$ soles mensuales. Este bajo valor revela la poca importancia y poco conocimiento de la existencia de dicha laguna por parte de los usuarios (Lucich \& Gonzáles, 2015). En cambio, la variable recuperación y conservación de otras lagunas, abandonando la recuperación de la laguna de Huacracocha, revela que el bienestar de los usuarios disminuye en S/.2,88 soles mensuales; en este caso, el costo de oportunidad de buscar nuevas fuentes de agua superficial estaría asociado a la pérdida de los servicios ecosistémicos hídricos, biodiversidad y belleza escénica (Wunder, 2006). De ahí que, pasar de la estrategia de intervención basada en la recuperación de otras lagunas (ROL) a otra basada en recuperar y conservar los servicios ecosistémicos de la laguna de Huacracocha $(\mathrm{RLH})$, dejando constante los demás atributos del sistema del servicio de agua potable, aumentaría el bienestar Hicksiana en S/.4,53 soles mensuales (Tabla 4, opción de política II), reflejando prioridad dicha estrategia y compatible con lo manifestado en el análisis descriptivo.

Lucich \& Gonzáles (2015), identificaron algunas razones del rechazo de los usuarios al traer agua de otras fuentes como: el mayor costo de inversión que redundaría en las tarifas, la inviabilidad técnica o no sostenible y el deterioro de la actual fuente; conllevando que, en el futuro, se tenga que enfrentar la misma problemática de escasez de agua y por consiguiente, menos horas de continuidad del servicio de agua potable

Esto es, los cambios monetarios del bienestar ordenados según estados u opciones de política (Tabla 4) a partir de agregar todos o parcialmente los valores marginales de cada atributo, considerando las mejoras del sistema del servicio de abastecimiento de agua potable en condiciones de seguridad hídrica, debido a las estrategias de intervención de los planes de mejora de cada 
Tabla 4

Cambio en el bienestar

económico por opciones de política pública

\begin{tabular}{|c|c|c|c|c|c|c|}
\hline \multirow[b]{2}{*}{ Servicios de agua potable (atributos) } & \multicolumn{3}{|c|}{ Coeficientes por niveles de mejora } & \multicolumn{3}{|c|}{ Cambios monetarios en el bienestar } \\
\hline & Deficiente* & Bueno & Excelente & $\begin{array}{l}\text { Opción de } \\
\text { política I }\end{array}$ & $\begin{array}{l}\text { Opción de } \\
\text { política II }\end{array}$ & $\begin{array}{l}\text { Opción de } \\
\text { política III }\end{array}$ \\
\hline Continuidad del agua potable $12 \mathrm{H}$ & -0.2022 & 0.2022 & & 1.9862 & & \\
\hline Continuidad del agua potable $24 \mathrm{H}$ & -0.7623 & & 0.7623 & & 7.4864 & 7.4864 \\
\hline Disponibilidad fuente del agua ROL & 0.2928 & -0.2928 & & -2.8753 & -2.8753 & \\
\hline Disponibilidad fuente del agua RLH & -0.1684 & & 0.1684 & & 1.6538 & 1.6538 \\
\hline Tiempo de atención conexiones nuevas 10D & -0.3190 & 0.3190 & & 3.1326 & 3.1326 & \\
\hline Tiempo de atención conexiones nuevas 5D & -0.7972 & & 0.7972 & & & 7.8297 \\
\hline Total & -1.9563 & 0.2284 & 1.7279 & 2.243 & 9.397 & 16.970 \\
\hline
\end{tabular}

* Multiplicando por -1 la suma de los coeficientes de cada nivel de cada atributo se obtuvo la "utilidad marginal" del nivel "Deficiente" o statu quo del atributo.

alternativa; se estimaron, reemplazando los coeficientes de la ecuación de la función de utilidad indirecta (5) en la ecuación de la variación compensatoria (4), evidenciaron que, un estado o escenario óptimo (Opción de política III), donde: la continuidad del agua se asegura por 24 horas al día; recuperación y conservación de los servicios de regulación hídrica de la laguna de Huacracocha y una reducción a cinco días en el tiempo de atención en conexiones nuevas, inciden favorablemente en un cambio de bienestar económico del orden de S/ . 16,97 soles por usuario mes.

En cambio, un estado o escenario apuesta (Opción de política II), caracterizado por: continuidad del agua durante 24 horas por día; recuperación y conservación de los servicios de regulación hídrica (disposición a aceptar o compensación potencial); recuperación y conservación de los servicios de regulación hídrica de la laguna de Huacracocha; y reducción del tiempo de atención de conexiones nuevas a 10 días; impactan, favorablemente en el cambio del bienestar económico en el orden de S/ 9,40 soles por usuario mes, a diferencia de un estado de escasa eficiencia operacional o escenario tendencial (Opción de política I), que significa un cambio del bienestar económico de solo S/. 2,24 soles por usuario mes.

Es necesario recalcar que, si consideramos en al análisis solo los tipos de fuentes de agua, a través de la estrategia de intervención consistente en traer agua de las otras nueve lagunas (ROL), por la estrategia de recuperar y conservar la laguna de Huacracocha (RLH), sin cambios en los demás atributos, el bienestar de los usuarios mejora en $\mathrm{S} / .4,53$ soles mensuales. Así mismo, una estrategia de intervención que implica un aumento de 12 de horas de servicio de agua potable a 24 horas por día, genera una mejora en el bienestar de $\mathrm{S} / .5,50$ soles mensuales. Además, un cambio de intervención que implica una reducción del tiempo de atención de conexiones nuevas de agua potable de 10 días a un estándar de cinco días, genera un cambio en el bienestar de S/.4,70 soles mensuales.

Por otro lado, el atributo tiempo de atención de conexiones nuevas del agua potable y la reducción del mismo de más de quince a cinco días, fue preferido y valorado por los usuarios con $\mathrm{S} / .3,91$ soles mensuales. En particular, la SUNASS (2016), en esta variable, calificó a la EPS como de desempeño "Malo", debido a que el $50 \%$ de solicitudes nuevas pagadas por el cliente fueron atendidas con más de 15 días hábiles (estándar $100 \%=15$ días hábiles); en cambio, el desempeño de SEDA AYACUCHO S.A. fue calificado como de "Buena práctica", debido a que contrató la ejecución de las conexiones nuevas mediante la tercerización con una empresa de servicios, a quienes ha puesto como plazo realizarla en cinco días. El contrato incluyó la calidad de la ejecución como un factor que puede originar una penalidad para el contratista (SUNASS 2016). Por tanto, una estrategia de intervención que reduzca el tiempo de atención de quince, o más días, a diez días y, de esta a cinco días, la disposición marginal a pagar es de $\mathrm{S} / .2,34$ soles mensuales, que equivalen a un aumento del bienestar Hicksiana del orden de S/.4,70 soles mensuales (Opción de política III y política II).

Con respecto a las características socioeconómicas de los usuarios domésticos del sistema de servicio de agua potable, se evidencia que la probabilidad de que estos elijan alguna estrategia de intervención de los planes de mejora disminuye a medida que aumenta su edad, en cambio, se infiere que los jóvenes están a favor de estas mejoras (Carbajal \& Lucich, 2016). Así mismo, es menos probable que los usuarios que actualmente registran elevados pagos por su servicio de agua potable puedan estar dispuestos a realizar pagos adicionales para acceder a algún plan de mejora. Aunque, en 
la medida en que el ingreso familiar aumenta, la probabilidad de elegir los planes de mejora crece (Lucich \& Gonzáles, 2015; Ojeda, et al., 2017). En consecuencia, es más probable que hogares con mayores ingresos acepten planes, proyectos o acciones de mejora de los niveles de cada atributo del servicio de agua potable, que aquellos hogares con menores ingresos.

Es necesario recalcar que el género, no incide sobre la elección de las alternativas o planes de mejora; sin embargo, Carbajal \& Lucich (2016), argumentaron que las mujeres son menos propensas a elegir planes de mejora, pero están dispuestas a pagar S/.1,40 soles mensuales menos que los hombres (Scarpa, et al., 2012), y valoran más la conservación de la fuente del agua. Así mismo, la actividad económica del jefe del hogar no incide en la elección de los usuarios por planes de mejora, muy en línea con lo encontrado por Lucich \& Gonzáles (2015). Por otro lado, se evidencia que hay indicios que hogares con mayor número de integrantes son proclives a favor de planes, proyectos o acciones de mejora de los atributos del servicio de agua potable.

Igualmente, hay indicios que la educación de los usuarios incide en la elección de planes de mejora de los atributos del servicio de abastecimiento de agua potable. Carbajal \& Lucich (2016), afirman que conforme se incrementa el nivel educativo, se incrementa la utilidad de los usuarios por acceder a un plan, proyecto o acción de mejora. Un público educado a menudo entiende la idea de pago por servicios ecosistémicos hídricos, "siente una conexión hacia ella, y puede ser motivado a reconocer que hay que hacer inversiones para poder seguir disfrutando cuencas saludables y productivas" (Asquith \& Wunder, 2009).

En definitiva, al agregar la DAPMg por las mejoras en cada nivel de los atributos del sistema del servicio de abastecimiento de agua potable como: continuidad del agua potable por 24 horas día, disponibilidad de fuentes de agua priorizando la recuperación y conservación de la laguna de Huacracocha y, tiempo de atención de conexiones nuevas en 10 días; los usuarios domésticos están dispuestos a pagar S/.9,61 soles por mes vivienda, muy en línea con la medida monetaria del cambio del bienestar Hicksiana del orden de S/.9,40 soles por mes usuario (opción de política II).

También se evidenció, la prioridad de los atributos continuidad del agua y tiempo de atención de conexiones nuevas de agua potable debido a que son los más valorados; por tanto, cualquier política, a través de estrategias de intervención, debe solucionar el problema de oferta de agua potable desde las fuentes de agua, con infraestructura natural, infraestructura de producción y distribución, complementadas con el logro de la eficiencia operativa a través del personal técnico capacitado o de tercerización del servicio de conexiones de la EPS. Así mismo, esta opción de política o planes de mejora está fuertemente condicionada por la edad y nivel de ingreso familiar de los usuarios domésticos del área metropolitana de Huancayo.

Aunque, el estudio actual tenga fortalezas es posible que se muestran limitaciones, por realizarse con una muestra de jefes de hogar, que incluyó tarjetas de elección en un solo instrumento para todos los sectores del área de estudio. Así mismo, las decisiones de elección manifestadas pueden diferir de las efectivamente elegidas al momento de ejecutar los planes de mejora. Por lo que, se necesitan nuevos estudios con diseños longitudinales y/o experimentales que generan una sucesión de teorías afinadas progresivamente y con el objetivo de seguir ganando precisión empírica (Ortegón, 2015).

A pesar de que se necesitan más estudios, se sugiere a la EPS o a los tomadores de decisiones, diseñadores de políticas públicas regionales o locales, diseñadores de estructuras tarifarias que incorporan pagos por servicios ecosistémicos, evaluadores costo-beneficio de los planes de mejora, proyectos o estrategias de intervención en componentes que generan mayor bienestar; incorporar el valor económico encontrado para cada nivel de los atributos del sistema de servicios de abastecimiento de agua potable y el valor monetario del bienestar de los usuarios domésticos, agregando estos beneficios económicos en función de la población directamente beneficiaria.

\section{Conclusiones}

- Los usuarios domésticos están dispuestos a pagar S/ .9,61 soles por mes vivienda al agregar la DAPMg por las mejoras en cada nivel de los atributos del sistema del servicio de abastecimiento de agua potable.

- Cualquier política, a través de estrategias de intervención, debe solucionar el problema de oferta de agua potable desde las fuentes de agua, complementadas con el logro de la eficiencia operativa a través del personal técnico capacitado o de tercerización del servicio de conexiones de la EPS.

- Hay indicios que la educación de los usuarios incide en la elección de planes de mejora de los atributos del servicio de abastecimiento de agua potable.

- La actividad económica del jefe del hogar no incide en la elección de los usuarios por planes de mejora.

- Se evidencia que hay indicios que hogares con mayor número de integrantes son proclives a favor de planes, proyectos o acciones de mejora de los atributos del servicio de agua potable. 


\section{Referencias bibliográficas}

Adamowicz, W., Boxall, P., Williams, M., \& Louviere, J. (1998). Stated preference approaches for measuring passive use values: Choice experiments and contingent valuation. American Journal of Agricultural Economics, 80(1), 64-75. https://doi. org/10.2307/3180269

Alpízar, F., Carlsson, F., \& Martinsson, P. (2001). Using Choice Experiments for Non-Market Valuation Francisco Alpízar, Fredrik Carlsson 1 and Peter Martinsson. Economic Issues, 8, 83-110.

Asquith, N., \& Wunder, S. (2009). Pagos por servicios hídricos: las conversaciones de Bellagio. Fundación Natura Bolivia: Santa Cruz de La Sierra, 36.

Banco Interamericano de Desarrollo-BID. (2015). Liderando el desarrollo sostenible de las ciudades (Semana 5).

Banco Interamericano de Desarrollo-BID. (2016). Huancayo: Hacia la sostenibilidad metropolitana bajo el liderazgo de un gobierno local moderno. Miraflores-Perú.

Carbajal, N. M. A., \& Lucich, L. I. M. (2016). Valor de la conservación de la fuente de agua y de los atributos del servicio de abastecimiento de agua de SEDACusco: Una aproximación empleando experimentos de elección. Lima Perú.

Carlsson, F., Frykblom, P., \& Liljenstolpe, C. (2003). Valuing wetland attributes: an application of choice experiments. Ecological Economics, 47(1), 95-103. https://doi.org/10.1016/j.ecolecon.2002.09.003

Cerda, C. (2011). Una aplicación de experimentos de elección para identificar preferencias locales por opciones de conservación y desarrollo en el extremo sur de Chile. Bosque (Valdivia), 32(3), 297-307. https://doi.org/10.4067/S0717 92002011000300011

EPS SEDAM HUANCAYO. (2014). Plan Maestro Optimizado Segundo Quinquenio 2014-2018.

Farreras, V. (2014). Valoración económica de los efectos de la presión antrópica sobre el piedemonte mendocino. Una aplicación de los experimentos de elección discreta. Rev. FCA UNCUYO, 46(2), 113-133.

Hausman, J., \& MaFadden, S. (1984). Specification tests for the multinomial logit model. Econometrica, 52(5 (Sep.,1984)), 1219-1240.

Hensher, D. A., Rose, J. M., \& Greene, W. H. (2005).
Applied choice analysis. A Primer. Cambridge University Press, (Vol.53). Cambridge University Press.https: / / doi.org/10.1017/CBO9781107415324.004

Hensher, D., Shore, N., \& Train, K. (2005). Households' willingness to pay for water service attributes. Environmental and Resource Economics, 32(4), 509-531. https://doi.org/10.1007/ s10640-005-7686-7

Hjerpe, E. E., \& Hussain, A. (2016). Willingness to pay for ecosystem conservation in Alaska's Tongass National Forest: A choice modeling study. Ecology and Society, 21(2). https://doi.org/10.5751/ES08122-210208

Holmes, T. P., \& Adamowicz, W. L. (2003). Attribute-based methods. Forest Service, Southern Research Station, and University of Alberta.

Iglesias, P. D. (2017). La valoración económica y mercantilización del agua de consumo humano en el Estado de México. Algunos determinantes. Espiral Estudios Sobre Estado y Sociedad, Enero, XXIV(68), 79-110.

Justes, A., Barberán, R., \& Farizo, B. A. (2014). Economic valuation of domestic water uses. Science of the Total Environment, 472, 712-718. https://doi. org/10.1016/j.scitotenv.2013.11.113

Koehler, J., Rayner, S., Katuva, J., Thomson, P., \& Hope, R. (2018). A cultural theory of drinking water risks, values and institutional change. Global Environmental Change, 50(November 2017), 268-277. https://doi.org/10.1016/j.gloenvcha.2018.03.006

Lancaster, K. J. (1966). A new approach to consumer theory. The Journal of Political Economy, 74(2), 132-157. https://doi.org/10.3390/molecules14114337

Latinopoulos, D. (2014). Using a choice experiment to estimate the social benefits from improved water supply services. Journal of Integrative Environmental Sciences, 11, 187-204. https://doi.org/10.1080/1943815X.2014.942746

Lucich, L., I., \& Gonzales, K., K. (2015). Valoración económica de la calidad y confiabilidad de los servicios de agua potable en Tarapoto a través de experimentos de elección. https: / / doi.org/10.1002/ POLA

McFadden, D. (1974). Conditional logit analysis of qualitative choice behaviour. In en P. Zarembka (Ed.), Frontiers in econometrics (pp. 105-142). Retrieved from https://eml.berkeley.edu/reprints/mcfadden/zarembka.pdf 
Merino-Castelló, A. (2003). Eliciting consumers preferences using stated preference discrete choice models: Contingent ranking versus choice. UPF Economics and Business Working Paper. Universitat Pompeu Fabra, Ramon Trias Fargas 25-27, 08005 Barcelona, Spain. Retrieved from http:// papers.ssrn.com/sol3/papers.cfm?abstract_ $\mathrm{id}=562982 \% 5$ Cnhttp: / /ideas.repec.org / p/upf / upfses $/ 705 . \mathrm{html}$

Ministerio del Ambiente-MINAM. (2015). Manual de valoración económica del patrimonio natural. Lima Perú. Retrieved from http://www.minam. gob.pe/patrimonio-natural/wp-content/uploads/ sites/6/2013/09/MANUAL-VALORACIÓN-1410-15-OK.pdf

Ministerio del Ambiente-MINAM. (2016). Guía de valoración económica del patrimonio natural. Lima Perú.

Monroy, R., Valdivia, R., Sandoval, M., \& Rubiños, J. E. (2011). Valoración económica del servicio ambiental hidrológico en una reserva de la biosfera. Terra Latinoamericana, 29(3), 315-323.

Ojeda De La Cruz, A., Álvarez, C. R., Ramos, M., \& Soto, F. (2017). Determinants of domestic water consumption in Hermosillo, Sonora, Mexico. Journal of Cleaner Production. https://doi.org/10.1016/j.jclepro.2016.11.094

Ortegón, E. (2015). Políticas públicas: métodos conceptuales y métodos de evaluación. Huancayo: Universidad Continental.

Sadoff, C., \& Muller, M. (2010). La gestión del agua, la seguridad hídrica y la adaptación al cambio climático: Efectos anticipados y respuestas esenciales. GlobalWater Partnership Comité Técnico (TEC), 14, 108.

Scarpa, R., Thiene, M., \& Hensher, D. A. (2012). Preferences for tap water attributes within couples: An exploration of alternative mixed logit parameterizations. Water Resources Research. https://doi.org/10.1029/2010WR010148

Scarpa, R., Thiene, M., \& Hensher, D. A. (2012). Preferences for tap water attributes within couples: An exploration of alternative mixed logit parameterizations. Water Resources Research, 48, 1-11. https://doi.org/10.1029/2010WR010148

Superintendencia Nacional de Servicios de Saneamiento-SUNASS. (2015). Estudio tarifario. Lima Perú.

Superintendencia Nacional de Servicios de Saneamiento-SUNASS. (2016). Benchmarking regulatorio de las EPS (Datos 2015). Lima Perú.

Tudela, Juan; Soncco, C. (2016). Valoración económica del servicio ambiental hidrológico de las lagunas del alto Perú, Cajamarca: una aplicación del método de valoración contingente y, (August), $380-381$.

Tudela, J., \& Leos, J. (2017). Herramientas metodológicas para aplicaciones del experimento de elección. México: Universidad de Chapingo. Retrieved from http: / / repositorio.chapingo.edu.mx:8080/handle $/ 20.500 .12098 / 269$ ?show $=$ full

Tudela Mamani, J. W., \& Leos Rodríguez, J. A. (2018). Estimation of economic benefits due to improvements in basic sanitation services through choice experiments. Revista Chapingo Serie Ciencias Forestales y del Ambiente, 24(2), 237-250. https://doi. org/10.5154/r.rchscfa.2017.05.037

Wunder, S. (2006). Pagos por Servicios Ambientales: principios básicos esenciales. CIFOR Occasional Paper, 42(3), 14 